\title{
Investigation of polymer interaction with nanoscale modifier and development of its identification algorithm
}

\author{
Gudkov Maksim \\ Voronezh Institute of State Firefighting Service of \\ EMERCOM of Russia \\ Voronezh, Russia \\ Gudokmaksim@yandex.ru \\ Glotova Irina \\ Faculty of Technology and Merchandizing \\ Voronezh State Agrarian University \\ Voronezh, Russia \\ glotova-irina@yandex.ru
}

\author{
Shakhov Sergey \\ Faculty of food machines \\ Voronezh State University of Engineering Technologies \\ Voronezh, Russia \\ s_shahov@mail.ru
}

\author{
Pugacheva Inna \\ Faculty of Ecology and Chemical Technology \\ Voronezh State University of Engineering Technologies \\ Voronezh, Russia \\ eco-inna@yandex.ru \\ Galochkina Nadezhda \\ Faculty of Technology and Merchandizing \\ Voronezh State Agrarian University \\ Voronezh, Russia \\ Galochkina.na@mail.ru \\ Bokadarov Stanislav \\ Voronezh Institute of State Firefighting Service of \\ EMERCOM of Russia \\ Voronezh, Russia \\ Galochkina.na@mail.ru
}

\author{
Lobacheva Natalia \\ Faculty of food machines \\ Voronezh State University of Engineering Technologies \\ Voronezh, Russia \\ naloni@mail.ru
}

\begin{abstract}
The purpose of the work is to substantiate the mechanism of interaction of a mixture of fullerene fractions $\mathrm{C}_{50 \div 92}$ with the polymer matrix of natural and synthetic rubbers with the subsequent solution of the task of identifying their compliance with specified technological parameters in terms of the content of a nanoscale component. The authors make a study of an industrial brand of synthetic butadiene rubber: SBR-Nd and SBR-Ti, according to synthesize on neodymium and titanic catalysts such as natural rubber grade NR RSS1. As a modifier of the polymer matrix, a mixture of fullerenes of fraction $\mathrm{C}_{50}-\mathrm{C}_{92}$ was used in the following ratio: $\mathrm{C}_{50}-\mathrm{C}_{58}(14.69 \%), \mathrm{C}_{60}(63.12 \%)$, $\mathrm{C}_{62}-\mathrm{C}_{68}(5.88 \%), \mathrm{C}_{70}(\mathbf{1 3 . 2 5 \%}), \mathrm{C}_{72}-\mathrm{C}_{92}(3.06 \%)$. When processing the spectra, we used: autofiltration of the signal by a running spectral window, automatic correction of the baseline, complicated ATR correction for the depth of penetration into the film material. It is revealed that the nature of the interaction of a mixture of fullerenes with rubbers is determined by the uncertainty and chemical nature of the elastomer and does not depend on the nature and activity of the filler. An algorithm is developed for the identification of diene rubbers modified with a mixture of fullerenes. Practical approbation of the algorithm on the example of identification of a mixture of $\mathrm{C}_{50}-\mathrm{C}_{92}$ fullerenes in
\end{abstract}

polymer matrices of various chemical nature is carried out based on the IR spectroscopy method. The accuracy of identification was $97-98 \%$.

Keywords-natural rubber, polybutadiene, fullerene, nanocomposites, discriminant analysis, IR spectroscopy

\section{INTRODUCTION}

The use of biopolymer nanocomposites in various industries allows to increase production efficiency and improve the functional characteristics and quality indicators of products. The introduction of nanocomponents in the polymer matrix makes it possible to obtain structural and biomaterials with increased heat resistance, improved barrier and mechanical properties [1-4].

The use of nanomaterials in the packaging of food products allows the creation of barrier materials based on biomodified collagen, chitosan, latex coatings modified with nano-silver particles, and also informing about the state of products (biochips, nanochips, biosensors) [5-7]. The use of 
nanoclay, carbon nanotubes as fillers leads to a decrease in the apro- and aromatic permeability of the package [8].

Nonmetallic construction materials are in great demand in engineering, including the protection of metal structures from corrosion. Their use is also beneficial from the economic point of view, since it allows replacement of more expensive materials based on metals.

Promising construction materials include polymers and polymer composites that have nanoscale particles in their structure - fullerenes and other carbon nanomaterials. It is shown that the mixture of fullerenes of the $\mathrm{C}_{50}-\mathrm{C}_{92}$ fraction is a complex modifier of the properties of NR RSS1, SBR-Ti and rubbers based on them [9]. A one and a half to two - fold increase in the resistance to repeated stretching and heat aging of rubbers based on NR RSS1 and SBR-Ti modified with a mixture of fullerenes with the following brands of carbon black: N330, N375, N550 was established [10]. It is necessary to study the mechanisms of interaction of nanoscale modifiers with elastomers of different structures

Research results shows that the mechanism of interaction of a mixture of fullerenes and fullerene-containing technical carbon (FTC) with polymers does not fit into the framework of the classical theory of amplification (strengthening). The modifying activity of the mixture of fullerenes is in the field of micro doses, probably it is connected with the developed specific surface area $\left(600 \mathrm{~m}^{2} / \mathrm{g}\right.$ for $\mathrm{C}_{60}$ fraction), the value of which exceeds all existing fillers and elastomers modifiers [9, $10]$.

The purpose of the work is to substantiate the mechanism of interaction of a mixture of $\mathrm{C}_{50}-\mathrm{C}_{92}$ fullerenes with a polymer matrix of natural and synthetic rubbers with the subsequent solution of the problem of identifying their correspondence to the specified technological parameters in terms of a nanoscale component content.

\section{MATERIALS AND METHODS}

Industrial brand of synthetic butadiene rubber SBR-Nd and SBR-Ti, according to synthesize on neodymium and titanic catalysts such as natural rubber grade NR RSS1 were used as objects of research. A mixture of fullerenes of fraction $\mathrm{C}_{50}$ $\mathrm{C}_{92}$ in the following ratio was used as the polymer matrix modifier: $\mathrm{C}_{50}-\mathrm{C}_{58}(14.69 \%), \mathrm{C}_{60}(63.12 \%), \mathrm{C}_{62}-\mathrm{C}_{68}(5.88 \%)$, $\mathrm{C}_{70}(13.25 \%), \mathrm{C}_{72}-\mathrm{C}_{92}(3.06 \%)$.

Twenty four samples of polymer rubber films on an aluminum foil substrate were investigated.

IR Fourier spectroscopy was used in the variant with 10fold beam passage through the sample and an attenuated total refection (ATR) crystal (NICOLET6700).

The processing of the spectra included: autofiltration of the signal by a running spectral window, automatic correction of the baseline, complicated ATR correction for the depth of penetration into the film material.

The processing of the results of the studies was carried out in accordance with the accepted statistical methods and criteria for checking for adequacy.

\section{RESULTS AND DISCUSSION}

The authors [11, 12] divide the compositional materials into groups:

- materials containing a nanocomponent in the bulk;

- materials containing nanocomponent intermediate structures;

- $\quad$ layered (laminated) materials;

- the materials containing a nanocomponent on a surface.

The analysis shows that when developing methods for obtaining nanocomposite materials, it is necessary to take into account the high surface energy and the small size of the nanocomponents introduced. When polymeric composites are modified with nanoscale fillers, the problem of their precise identification arises due to the fact that the concentration of the modifier is not large and it is not always possible to detect their presence in a multicomponent mixture by conventional methods. In addition, in the technological process of production of filled composites, it is necessary to control the dosing process (to ensure the accuracy of application and to maintain optimum concentrations to achieve a particular property of the composition).

To solve such problems, the authors used IR-spectroscopy, and the authors - together with discriminant analysis.

Discriminant analysis is used to decide which variables distinguish two or more emerging sets (groups). Further, if these variables are known, for a new experiment it is possible to predict which group the new object under study belongs to.

When polymeric composites are modified with nanoscale fillers, the problem of their precise identification arises due to the fact that the concentration of the modifier is small and conventional methods do not always manage to identify their presence in a multicomponent mixture. In addition, in the technological process of production of filled composites, it is necessary to control the dosing process (to ensure the accuracy of application and to maintain optimum concentrations to achieve a particular property of the composition).

The task of technology conformity identifying is often met in various applied research. In particular, one of the approaches to solving the current problem [13, 14] is a twostage analysis method, according to which in the first stage a preliminary determination of the substance nature is carried out, and then the final determination of polymers or oligomers structure by the methods of qualitative and quantitative analysis takes place. This approach can undoubtedly be attributed to the classical one due to the fact that the proposed methods are mentioned in the earlier edition [15]. The disadvantage of the proposed methods of identification is that they do not suppose the use of modern computer technology. Its emergence and widespread use in statistical research not only made it possible to accelerate and facilitate the identification procedure significantly, but also created the potential to use more complex mathematical methods together with promising methods (NMR, Mass spectrometry, FourierIR spectroscopy). 
Depending on the identification task complexity, researchers suggest the use of one method of Fourier-IRspectroscopy or thermogravimetry with subsequent computer data processing [16-18]. There is a number of disadvantages in the algorithm operation:

1) there are no neglects and tolerances in multicomponent analysis;

2) the formation of uncharacteristic peaks caused by intermolecular interaction results into difficulties in searching with spectra from a data bank.

The use of promising methods makes it possible to identify crystalline and partially crystalline polymeric materials, which was a challenging task until now [19].

To determine the changes in the vibrations, the Fouriertransform spectroscopy method was used in the structure of the investigated rubbers in a variant with a 10 -fold beam passage through the sample, in the range from 400 to 4000 $\mathrm{cm}^{-1}$. Samples of rubbers were monolithic films with a thickness of $20 \mu \mathrm{m}$, obtained on an aluminum substrate from $1 \%$ of toluene solutions. Oxidation of the films was carried out in a low-temperature laboratory electric furnace "SNOL $58 / 350 "$ at a temperature of $100^{\circ} \mathrm{C}$ for 24 hours. The results are shown in Fig. 1.
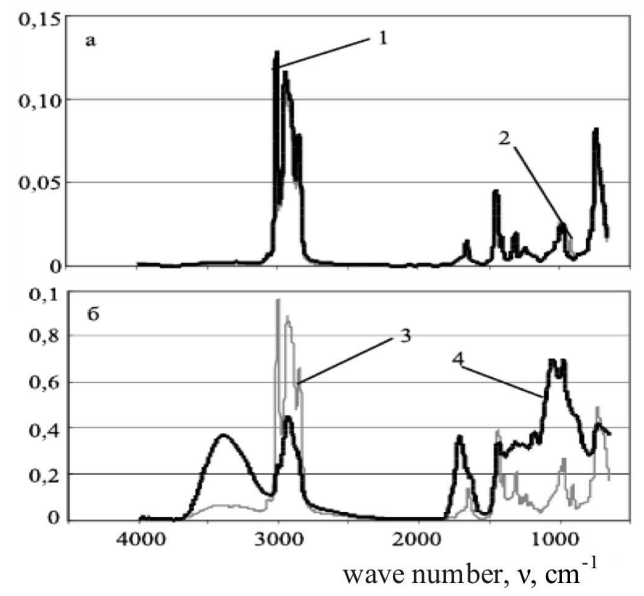

Fig. 1. IR spectra presidency rubbers: a - before the introduction of a mixture of fullerenes; $b$ - after modification: 1 - SBR-Nd; 2- SBR-Ti; 3 SBR-Ti modified by a mixture of fullerenes 0.015 mass shares; 4 - SBR-Nd modified by a mixture of fullerenes 0.015 mass shares

The spectra shown in Fig. 1a, practically coincide with each other except for the peak of $915 \mathrm{~cm}^{-1}$, which shows the ratio of cis-trans units in polybutadiene synthesized on a titanium catalyst. After modification with a mixture of fullerenes, the SBR-Nd rubber, in contrast to the SBR-Ti, is partly oxidized without preliminary heat treatment (Fig. 1b). This is confirmed by the appearance and expansion of absorption bands at 3450 and $1720 \mathrm{~cm}^{-1}$, which corresponds to the oscillation of hydroxyl $(-\mathrm{O}-\mathrm{H})$ and carbonyl $(-\mathrm{C}=\mathrm{O})$ groups, respectively.

SBR-Nd rubber, after heat treatment is subjected to oxidation, as evidenced by the presence of absorption bands of 3450 and $1720 \mathrm{~cm}^{-1}$, indicating the addition of oxygen in the form of hydroxyl and carbonyl groups (Fig. 2a). Oxidation of the investigated rubbers in a mixture with fullerenes occurs almost completely (Fig. 2b).
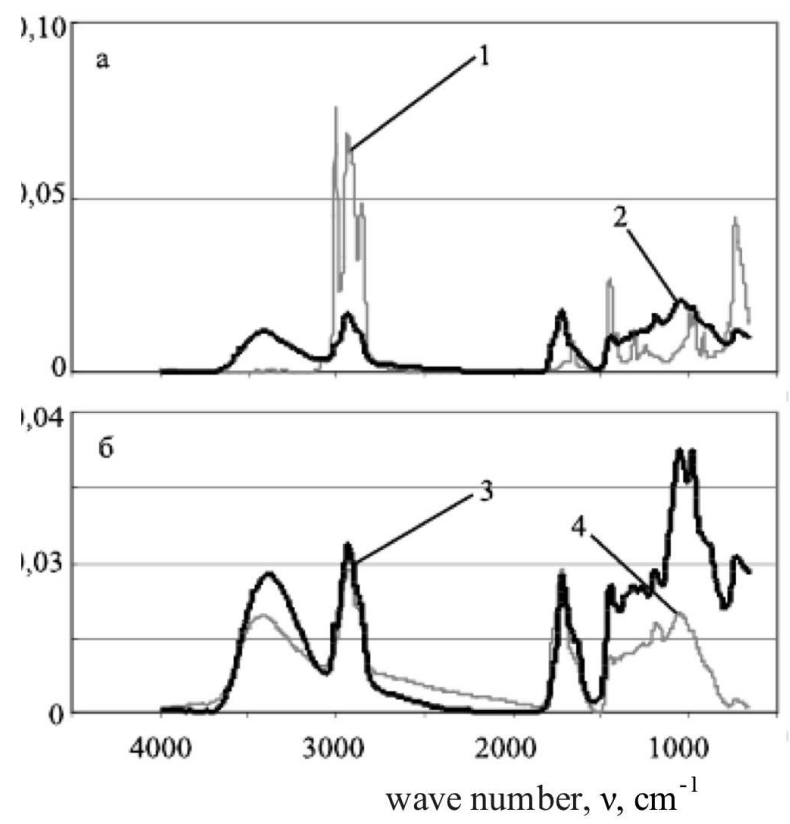

Fig. 2. IR spectra of oxidized in the air at $100^{\circ} \mathrm{C}$ for $24 \mathrm{~h}$ of re-precipitated rubbers: a - before the introduction of a mixture of fullerenes; $\mathrm{b}$ - after modification: 1-SBR-Ti; 2 - SBR-Nd; 3 - SBR-Nd modified with 0.015 mass shares of a mixture of fullerenes; 4 -SBR-Ti modified with 0.015 mass shares of a mixture of fullerenes

To obtain additional information from the results of the experiment on the methods considered above, an additional introduction of discriminant analysis is proposed by us. This will allow examining chemical interactions (if any) in detail. The method of discriminant analysis will be considered with the example of modified and processed polymers.

Discriminant analysis is used to decide which variables distinguish two or more emerging sets (groups). Further, if these variables are known, you can predict which group the new object being studied belongs to for a new experiment.

Let's try to make discrimination on the basis of the spectra portions comparison related to the characteristic IR absorption bands of individual functional groups and groups of atoms and types of bonds. To do this, we supplement the matrix of the initial data with a new categorical variable under the conditional name "Deformations and pi-bonds", bearing in mind that the system can interact with modifiers with pielectron systems and steric effects are possible for the deformation type of vibrations in molecules.

Let us briefly consider the conceptual apparatus of discriminant analysis that makes up its metric.

The criterion for the variable high discriminative ability is the Wilks criterion $l W$, which is defined as the ratio of the 
determinants of the variance-covariance matrices of the intragroup $\mathrm{W}$ and the general $\mathrm{T}$.

$$
I W=\operatorname{det}(W) / \operatorname{det}(T)
$$

It is quite obvious that the smaller the Wilks 1 value, the greater the variable discriminative ability.

Another value characterizing the metric of discriminant analysis is a particular Wilks criterion $l p$, which is equal to the ratio $l W$ after introduction of a new variable in the stepwise discriminant analysis to $l W$ before introduction of this variable.

$$
l p=l W(\text { after inputting }) / l W(\text { before entering) }
$$

This ratio characterizes the multiplicative contribution of the new variable after its introduction into the discriminant analysis.

Acceptance of hypotheses in discriminant analysis is characterized by F-statistics of Rao:

$$
F=[(n-q) /(q-1)] \times[(1-l p) / p]
$$

where $n$ is the number of experiments; $q$ is the number of classification groups; $p$ is the number of variables.

The criterion $\mathrm{F}$ for introduction / removal of a variable is associated with the $l p$ test. The value of the F-statistic for a variable indicates its statistical significance in the case of discrimination between sets, i.e. it is a measure of the contribution of a variable to the prediction of membership in a set.

Significance level $p$. This level is related to the value of $F$ for removal. It should be noted that this value should be used with a certain precaution.

Tolerance level T. The value of the tolerance level is calculated as $1-R_{2}$, where $R$ is the multiple correlation coefficient for the corresponding variable with all the other variables in the model. Thus, tolerance is a measure of the redundancy of the variable introduced into the analysis. For example, the $\mathrm{T}=0.10$ level means that the variable introduced into the model is $90 \%$ correlated with other model variables, i.e. it is redundant $\left(1-T=R_{2}\right)$. The value of tolerance is introduced into the analysis in connection with the problem of collinearity of variables. In this case, all calculations associated with poorly conditioned matrices that can not be accessed are impossible.

The classifying function $S_{i}$ is used to determine which class belongs to a particular sample (experiment). The number of classifying functions is equal to the number of the classification groups. Each function is used to calculate the score of the result for each sample (experiment) for each group and is calculated by the following formula:

$$
S_{i}=c_{i}+w_{i 1} \times x_{1}+w_{i 2} \times x_{2}+\ldots . .+w_{i m} \times x_{m},
$$

where the $i$ index refers to a group, indices $1,2, \ldots m$ refer to $m$ variables; $\mathrm{c}_{i}$ is the constant for $i$-th group, $\mathrm{w}_{i j}$ - statistical weights for the $j$-th variable when calculating the classification account (score) for the $i$-th group; $x j$ is the observed value in the corresponding experiment for the ${ }_{j}$-th variable.
The essence of discriminant analysis lies in the construction of a "model" in which one can predict which sample in the experiment which group belongs to. Here the term "in the model" is used for the included variables for prediction, and the term "outside the model" is applied to the variables that were not included in the model. In step-by-step discriminant analysis, all variables are looked through and it is determined which of the variables has the best discriminating ability between the groups.

The choice of the categorical variable "Deformations and pi-conjugation" will show us the results of changes in molecules vibrations under the modifiers action.

A small $\lambda$ Wilks value (at the level of 10-5) indicates a high discriminatory capacity of the spectrum segments chosen for analysis. Table 1 shows the categories of sections of the

\begin{tabular}{|c|c|c|c|c|c|}
\hline$\frac{\vec{t}}{\text { Z }}$ & $\frac{n}{2}$ & $\frac{2}{3}$ & 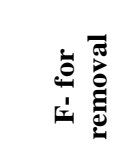 & $\mathbf{T}$ & 1-T \\
\hline № 1 & 0,000010 & 0,311984 & 83,2499 & 0,002772 & 0,997228 \\
\hline № 2 & 0,000009 & 0,357604 & 67,8136 & 0,002634 & 0,997366 \\
\hline № 4 & 0,000007 & 0,464259 & 43,5623 & 0,143617 & 0,856383 \\
\hline № 5 & 0,000007 & 0,453652 & 45,4636 & 0,003512 & 0,996488 \\
\hline № 6 & 0,000004 & 0,748637 & 12,6750 & 0,002200 & 0,997800 \\
\hline № 11 & 0,000006 & 0,548947 & 31,0180 & 0,007090 & 0,992910 \\
\hline \begin{tabular}{|l|} 
№ 20 \\
\end{tabular} & 0,000009 & 0,381621 & 61,1702 & 0,008461 & 0,991540 \\
\hline № 23 & 0,000020 & 0,165846 & 189,8706 & 0,000500 & 0,999500 \\
\hline № 24 & 0,000013 & 0,246905 & 115,1426 & 0,000510 & 0,999490 \\
\hline
\end{tabular}
spectrum of the rubber. Table 2 shows the classifying ability of selected spectral areas with characteristic oscillations.

TABLE I. SUMMARY RESULTS OF DISCRIMINANT ANALYSIS*

* Number of variables in the model - 9.Grouping variable - Deformations and $\pi$-links ( 5 groups). $\lambda$ Wilks - 0,0000. Approximating criterion Rao $F$ $(36,567)=443,67 . p<0.00005$. In the brackets of the criterion is the effective number of degrees of freedom. p-level $=0,000000$.

TABLE II. CLASSIFYING ABILITY OF SPECTRAL REGIONS BY TYPE OF OSCILLATION

\begin{tabular}{|l|l|l|}
\hline Group (oscillations types) & Wave number, $\mathbf{c m}^{-1}$ & $\begin{array}{c}\text { \% of correct } \\
\text { classification }\end{array}$ \\
\hline $\begin{array}{l}\text { Non-planar deformational } \\
\text { oscillations } \mathrm{CH}_{2} \text { in vinyl }\end{array}$ & from 904 to 995 & 100,0000 \\
\hline $\begin{array}{l}\text { Scissor oscillations } \mathrm{CH}_{2}, \\
\mathrm{CH}_{3}\end{array}$ & from 1406 to 1429 & 100,0000 \\
\hline $\begin{array}{l}\text { Valent oscillations } \mathrm{C}=\mathrm{C} \text { in } \\
\text { conjugated systems }\end{array}$ & from 1593 to 1649 & 100,0000 \\
\hline $\begin{array}{l}\text { Valence oscillations } \mathrm{C}=\mathrm{C} \\
\text { without conjugation }\end{array}$ & from 1650 to 1701 & 88,8889 \\
\hline $\begin{array}{l}\text { Valent oscillations of } \mathrm{C}-\mathrm{H} \\
\text { in } \mathrm{CH}, \mathrm{CH}_{2}, \mathrm{CH}_{3}\end{array}$ & from 2974 to 3061 & 100,0000 \\
\hline Totally & - & 98,1707 \\
\hline
\end{tabular}

From the analysis of Table 2, it follows that only the stretching vibrations of $\mathrm{C}=\mathrm{C}$ in a hydrocarbon chain without conjugation, possibly related to terminal vinyl groups or to monomer inclusions of alkenes, do not possess $100 \%$ predictive power.

The "bad (poor)" predictive ability of some characteristic oscillations in a group of samples in a chemical sense can mean that the effect of modifiers leads to the greatest data 
disperse due to the chemical interactions of the modifier with this group.

To study the effect of, for example, $\mathrm{X}$ and $\mathrm{Y}$ modifiers, it is sufficient to include them in a new categorical grouping variable of the type "Modifier type" containing the values "Modifier X", "Modifier Y", "No modifier", and to carry out a discriminant analysis with this variable. With a high discriminating ability, we can confidently conclude that the modifiers interact with the polymer base, as well as there is difference or similarity between the $\mathrm{X}$ modifier and the $\mathrm{Y}$ modifier.

On the basis of the experiment and calculations it was shown that the addition of a mixture of fullerenes to reprecipitated polybutadienes leads to an increase in the rate and completeness of their oxidation, especially with respect to the SBR-Nd. This is probably due to the interaction of terminal vinyl groups or monomer inclusions of alkenes with the introduced fullerenes.

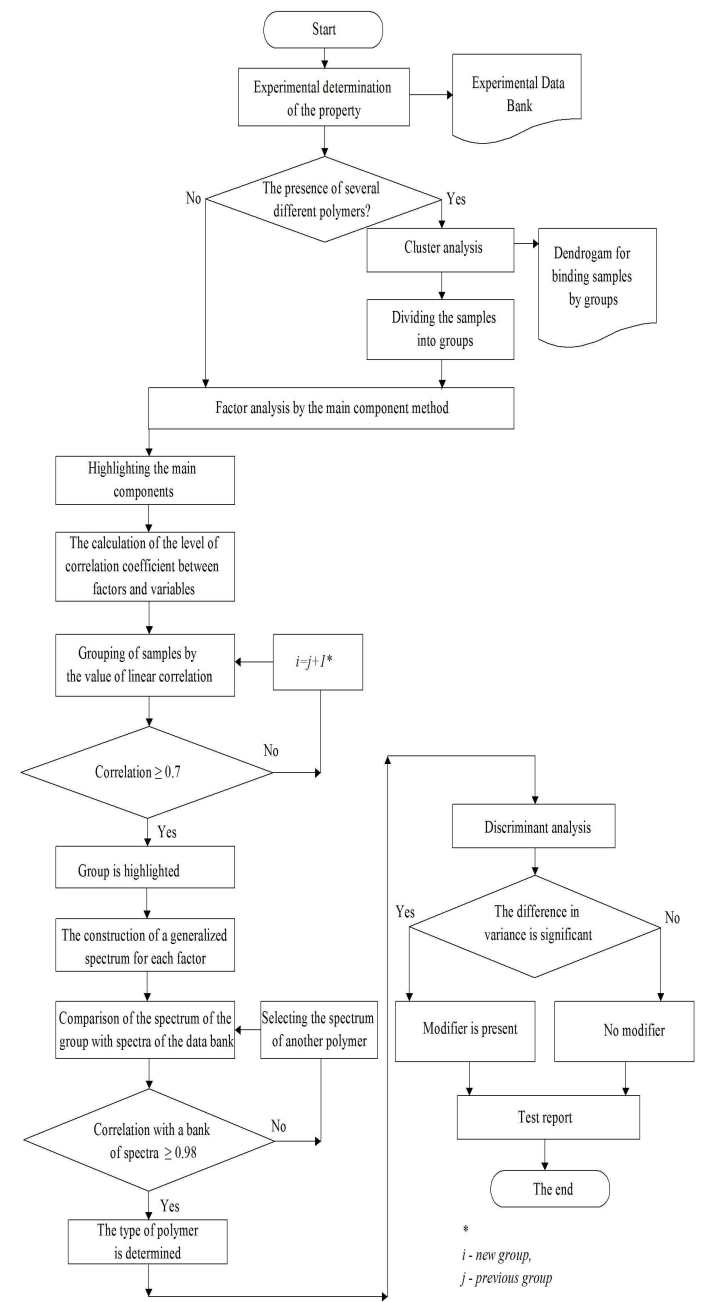

Fig. 3. Algorithm for identification of diene rubbers modified with a mixture of fullerenes

According to the IR analysis, the addition of oxygen is less intensive when fullerene is introduced into the polymer, in comparison with the initial state, the thermal stability increases, and various rubber bonds (especially scissors) are involved in the process, as indicated by the broadening of the peaks in the corresponding region. This allows us to conclude that fullerene attaches to itself polymer molecules over double bonds. Further interaction occurs according to the classical mechanism of chain radical decay.

To solve the problem of identification of diene rubbers modified by a mixture of fullerenes, an algorithm is proposed (Fig. 3). The method of identification of modified raw materials is based on statistical methods for processing experimental data. The test report on the presence of a modification is issued at the end of the testing procedure.

\section{CONCLUSIONS}

According to the experimental data of spectral analysis together with the discriminant analysis, a mechanism for the interaction of a mixture of fullerenes with fraction $\mathrm{C}_{50}-\mathrm{C}_{92}$ with the polymer matrix of the investigated rubbers is proposed. It was found out that the character of the interaction of a mixture of fullerenes with rubber components is determined by the uncertainty and chemical nature of the elastomer and does not depend on the nature and activity of the filler.

An algorithm for identifying the composition of diene rubbers modified with a mixture of fullerenes has been developed. Practical approbation of the algorithm on the example of identification of a mixture of $\mathrm{C}_{50}-\mathrm{C}_{92}$ fullerenes in polymer matrices of various chemical nature is carried out based on the IR spectroscopy method. The accuracy of identification was $97-98 \%$.

\section{References}

[1] S.S. Ray, M. Okamoto "Polymer/layered silicate nanocomposites: a review from preparation to processing," Progress in polimer science. vol. 28(11), pp. 1539-1641, 2003.

[2] C. Silvestre, D. Duraccio, S. Cimmino "Food packaging based on polymer nanomaterials," Progress in Polymer Science. vol. 36(12), pp. 1766-1782, 2011.

[3] A. Sorrentino, G. Gorrasi, V. Vittoria "Potential perspectives of bionanocomposites for food packaging applications," Trends in Food Science \& Technology. vol. 18(2), pp. 84-95, 2007.

[4] V.D. Timothy "Applications of nanotechnology in food packaging and food safety: Barrier materials, antimicrobials and sensors," Journal of Colloid and Interface Science. vol. 363(1), pp. 1-24, 2011.

[5] H. Bouwmeester, P. Brandhoff, H.J. Marvin, S. Weigel, R.J. Peters "State of the safety assessment and current use of nanomaterials in food and food production," Trends in Food Science \& Technology. vol. 40(2), pp. 200-210, 2014.

[6] S. Goyal, G.K. Goyal "Nanotechnology in food packaging a critical review," Russian Journal of Agricultural and Socio-Economic Sciences. vol. 10(10), pp. 14-24, 2012.

[7] A. LaCoste, K.M. Schaich, D. Zumbrunnen, K.L. Yam "Advancing controlled release packaging through smart blending," Packaging Technology and Science. vol. 18(2), pp. 77-87, 2005.

[8] A. Arora, G.W. Padua "Nanocomposites in food packaging," Journal of Food science. vol. 75(1), pp. 43-49, 2010.

[9] A.V. Chichvarin, T.I. Igumenova, M.A. Gudkov "Character of thermal destruction polybutadiene under the influence mix fullerene C50-C92 cluster," Fundamental investigations. No. 11-1, pp. 202-205, 2012.

[10] T.I. Igumenova, M.A. Gudkov, G.V. Popov "Study of the influence of fulleren-containing technical carbon in the combination with carbon of 
various activity on the properties of rubber," Industrial production and use of elastomers. No. 1, pp. 22-25, 2013.

[11] M. Alexandre, P. Dubois "P. Polymer-layered silicate nanocomposites: preparation, properties and uses of a new class of materials," Materials Science and Engineering: Reports. vol. 28(1), pp. 1-63, 2000.

[12] A. Piozzi, I. Francolini "Editorial of the special issue antimicrobial polymers," Int. J. Mol. Sci. № 14, pp. 18002-18008, 2013.

[13] T.S. Vydrina "Methods for identification of polymers," Textbook. Allowance. Ekaterinburg: the Urals State Forestry University, 2009, p. 92.

[14] D. Ponnamma, H.J. Maria, A.K. Chandra, S. Thomas "Rubber Nanocomposites: Latest Trends and Concepts," In: P. Visakh, S. Thomas, A. Chandra, A. Mathew (eds) "Advances in Elastomers II. Advanced Structured Materials," vol. 12, pp. 69-107, Springer, Berlin, Heidelberg, 2013

[15] J. Haslam, G.A.Willys "Identification and analysis of polymers," Moskow: Chemistry, 1971, p. 432.

[16] M. Ritz, E. Plevova, L. Vaculikova "Identification of clay minerals by infrared spectroscopy and discriminant analysis," Applied Spectroscopy. vol. 64(12), pp. 1379-1387, 2010.

[17] M. Ritz, L. Vaculikova, E. Plevova "Application of infrared spectroscopy and chemometric methods to identification of selected minerals," Acta Geodyn. Geomater., Vol. 8, No. 1 (161), pp. 47-58, 2011.

[18] D. Verma, K. Katti, D. Katti "Effect of Biopolymers on Structure of Hydroxyapatite and Interfacial Interactions in Biomimetically Synthesized Hydroxyapatite/Biopolymer Nanocomposites," Ann. Biomed. Eng., vol. 36, p. 1024, 2008.

[19] E. Shpilevsky, O. Penyazkov, S. Filatov, G. Shilagardi, P. Tuvshintur, D. Timur-Bator, D. Ulam-Orgikh "Modification of materials by carbon nanoparticles," Solid State Phenomena, vol. 271, pp.70-75, 2018. 